\title{
Is Edaravone the Future of Free Radical Scavenging in Inflammatory States?
}

Sir,

Free radicals in systemic circulation lead to several forms of diseases involving cardiovascular, cerebrovascular, and respiratory system. They are highly unstable compounds which lead to oxidative damage to cellular lipids, protein, and DNA. A lot of research has been done to investigate the role of free radicals in several diseases. ${ }^{[1]}$ This has led to the development of free radical scavengers. Free radical scavengers that are arbitrarily used in the practice of anesthesiology and critical care are ascorbic acid (Vitamin C), Vitamin E (beta carotene), alpha tocopherol, omega fatty acids, allopurinol, mannitol, multivitamin infusions, selenium. ${ }^{[2]}$ The failure of free radical model could be because it is introduced quite late during management, especially when the damage has occurred. Another reason could be, the dose has not been described by conducting well-designed studies.

Edaravone was developed as a potent free radical scavenger and has been widely used to treat acute ischemic stroke (AIS) in Japan since 2001. Use of edaravone as a neuroprotective agent after AIS is a Grade B recommendation in Japan, after lot of encouraging results derived from Japanese population. Hydroxyl radical-dependent and radical-independent lipid peroxidations are neutralized by edaravone which protects against free radical-related injuries following a cerebrovascular event. Edaravone is a low molecular weight agent having water- and lipid-soluble properties [Figure 1]. Due to such favorable properties, it easily crosses the blood-brain barrier. It also has antiapoptotic, antinecrotic, and anticytokine effects in

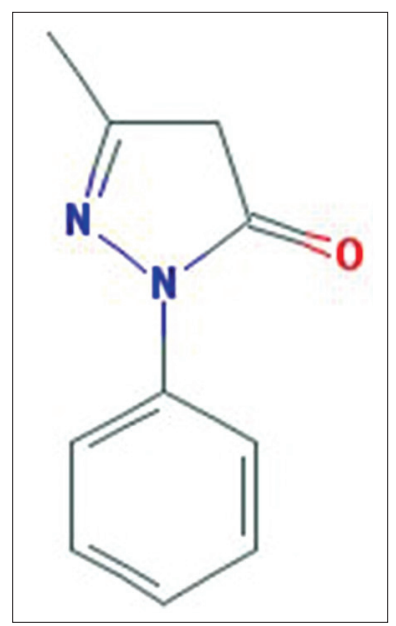

Figure 1: Chemical structure of edaravone (Image source: National Center for Biotechnology Information. PubChem Compound Database; CID =4021, https://pubchem.ncbi.nlm.nih.gov/compound/4021; accessed May 15, 2017) cardiovascular and cerebrovascular diseases. However, it is not supported by well-designed trials. In Japan, edaravone is used along with tissue plasminogen activator alteplase in patients with AIS. ${ }^{[3]}$ Kikuchi et al. reviewed edaravone and described it as a novel therapeutic intervention in diseases which are mediated through endothelial dysfunction such as atherosclerosis, heart failure, diabetes, hypertension as these diseases present with complications, and end organ damage due to oxidative stress with or without cytokine-induced apoptosis. The authors suggested that if edaravone infusion is timed properly in an acute coronary event or in life-threatening situations such as acute myocarditis, the free radical-induced myocardial injury might be prevented leading to a more viable myocardium at the end of the event. ${ }^{[4]}$ Elbaradey et al. used edaravone infusion in patients with septic peritonitis and compared it with a control group in which patients were treated with established management strategies. Patients in edaravone group received $30 \mathrm{mg} / 12 \mathrm{~h}$ for 2 weeks. They analyzed serum nuclear transcription factor kappa B activity, mitogen-activated protein kinase, heat shock protein 72 , and total antioxidant capacity. They found a significant decrease in inflammatory and oxidative markers in patients who received edaravone with a better clinical outcome compared to the control group. However, the sample size used by the researchers to arrive at the conclusion was less ( $n=30$ in each group). Although the researchers came out with convincing results, an adequately powered study with bigger sample size in future would be required to establish its efficacy in patients with septic peritonitis. ${ }^{[5]}$ Among the available free radical scavengers, edaravone appears to be the only one which has shown significant results in conditions which are mediated through free radical-induced oxidative damage. Edavarone is much cheaper than osagrel, another drug which is used to treat ischemic stroke. A vial of $20 \mathrm{ml}$, i.e., $30 \mathrm{mg}$, which is required for a day of treatment costs 500-600 rupees in Indian currency. More data from randomized controlled trials could establish the efficacy in conditions with multiple inflammatory pathways such as sepsis.

\section{Financial support and sponsorship}

Nil.

Conflicts of interest

There are no conflicts of interest.

Abhijit S. Nair

Department of Anesthesiology and Pain Management, Basavatarakam Indo American Cancer Hospital and Research Institute, Hyderabad, Telangana, India 
Address for correspondence: Dr. Abhijit S. Nair, Department of Anesthesiology and Pain Management, Basavatarakam Indo American Cancer Hospital and Research Institute, Hyderabad - 500 034, Telangana, India. E-mail: abhijitnair95@gmail.com

\section{References}

1. Hatwalne MS. Free radical scavengers in anaesthesiology and critical care. Indian J Anaesth 2012;56:227-33.

2. Pham-Huy LA, He H, Pham-Huy C. Free radicals, antioxidants in disease and health. Int J Biomed Sci 2008:4:89-96.

3. Kikuchi K, Miura N, Kawahara KI, Murai Y, Morioka M, Lapchak PA, et al. Edaravone (Radicut), a free radical scavenger, is a potentially useful addition to thrombolytic therapy in patients with acute ischemic stroke. Biomed Rep 2013;1:7-12.

4. Kikuchi K, Tancharoen S, Takeshige N, Yoshitomi M, Morioka M, Murai Y, et al. The efficacy of edaravone (radicut), a free radical scavenger, for cardiovascular disease. Int J Mol Sci 2013;14:13909-30.

5. Elbaradey GF, Elshmaa NS, Hodeib H. Role of edaravone in managemant of septic peritonitis. J Anaesthesiol Clin Pharmacol 2016;32:465-9.
This is an open access article distributed under the terms of the Creative Commons Attribution-NonCommercial-ShareAlike 3.0 License, which allows others to remix, tweak, and build upon the work non-commercially, as long as the author is credited and the new creations are licensed under the identical terms.

\begin{tabular}{|l|l|}
\hline \multicolumn{3}{|c|}{ Access this article online } \\
\hline Quick Response Code: & Website: \\
\hline & www.ijccm.org \\
\cline { 2 - 2 } & \\
\hline
\end{tabular}

How to cite this article: Nair AS. Is edaravone the future of free radical scavenging in inflammatory states? Indian J Crit Care Med 2017;21:475-6.

C 2017 Indian Journal of Critical Care Medicine | Published by Wolters Kluwer - Medknow 